\title{
A Peer-To-Peer Publication Model on Blockchain
}

\section{Imtiaz Khan * and Ali Shahaab \\ Cardiff School of Technologies, Cardiff Metropolitan University, Cardiff, United Kingdom}

In the past few decades, there has been a sharp rise of research irreproducibility and retraction, to a point that now is deemed as a crisis. Addressing this crisis, we present a peer-to-peer (P2P) publication model that utilizes blockchain and smart contract technologies. Focusing primarily on researchers and reviewers, the conceptual P2P publication model addresses the sociocultural and incentivization aspects of the irreproducibility crisis. In the P2P publication model, instead of a complete publication, a preapproved experimental design will be published on an incremental basis (unit-by-unit) and authorship will be shared with reviewers. The concept of the P2P publication model was inspired by the transformational journey the music publishing industry has undertaken as it traverses through vinyl age (complete albums) to the Spotify age (single-by-single), where there is a growing inclination among artists toward building an incremental album, taking account of feedback from fans and utilizing automated revenue collection and sharing systems. The ability to publish incrementally through the P2P publication model will relieve researchers from the burden of publishing complete and "good results" while simultaneously incentivizing reviewers to undertake rigorous review work to gain authorship credit in the research. The proposed P2P publication model aims to transform the century-old publication model and incentivization structure in alignment with open access publication ethos of the 21 st century.

Keywords: distributed ledgers, research reproducibility, scientific publishing, social factors, reward mechanism, blockchain

\section{INTRODUCTION}

For centuries, journals have remained the primary platform for scientific communication and act as a trusted third party to ensure the quality and integrity of published work. However, the paper and institutional subscription-based publication model established centuries ago has become unsustainable during the past few decades because of the growing popularity of the open access model where publications are online and authors pay for their publications. Correlated with this transformation, there is also a sharp rise of research irreproducibility and retraction to a point that now is deemed as a crisis across all disciplines (Baker, 2016). For example, in cancer research, a study found that 47 out of $53(\sim 90 \%)$ "landmark" research papers could not be reproduced (Begley and Ellis, 2012). Failure to reproduce scientific experiment not only undermines the "self-correcting" ethos of science but also limits the ability for science to progress. This is evident by our remarkably low ability to translate cancer research to clinical success despite extraordinary funding and public support for cancer research (Hutchinson and Kirk, 2011). The economic cost of irreproducibility is as high as $\$ 28 \mathrm{~B}$ per annum in the USA alone, where at least $50 \%$ of preclinical research remains irreproducible (Freedman et al., 2015). In the last few decades, retraction has also increased tenfold, 
with fraud accounting for $60 \%$ of these retractions (Brainard, 2018). High-profile retractions often have grave consequence on science and society. For example, in 2010, twelve years after publication, one of the world's oldest and most respected medical journal, The Lancet, retracted a paper that implied a link between the measles, mumps, and rubella (MMR) vaccine and autism (Eggertson, 2010). Based on this publication, tens of thousands of parents across the globe stopped using the MMR vaccination and as a result, measles returned as an endemic in Eastern and Northern Europe (Organisation, 2019). Seminal paper from Harvard economists that influenced Western governments to take austerity measures post 2008 global financial crisis was later found to have key errors that if found during review process would have an opposite impact on government's austerity policies (Krugman, 2013). Due to the austerity, millions of people became unemployed and tens of thousands lost their lives (Watkins et al., 2017). During the COVID-19 pandemic, just weeks after publication, the Lancet journal retracted a paper from Harvard professor that influenced the World Health Organization (WHO) and many other countries to halt hydroxychloroquine trials (James Heathers, 2020). Interestingly, it is not the peer-review process but rather investigative journalism or inquisitive student projects that found anomalies in these seminal publications. Aforementioned events therefore raise serious questions about the role of reviewers in publication processes, especially when human lives and public trust are at stake. In the long term, loss of trust will impact public perception toward science and eventually may constrain public funding for science.

Addressing this crisis, a number of initiatives have been undertaken to facilitate researchers to report and share detailed experimental methodologies in a standardised fashion along with policies to encourage or enforce researchers and journals to adhere to these standards. From a regulatory point of view, such initiatives may have resolved the technical and administrative issues associated with the crisis, but the success of attaining trust and sustainability of these initiatives hinges on the sociocultural contextualisation, strategic incentivization, and an answerability mechanism for all three primary stakeholders (researchers, reviewers, and journals) associated with the publication process.

In the 21st century, the currency of science is not only the quality but also the quantity of publication. Currently, researchers work within a "publish or perish" culture where they not only are under constant pressure of delivering a targeted number of publications but also are encouraged, if not compelled, to publish "good results" (Chambers, 2019). Therefore, the main focus of early to midcareer researchers in recent years has shifted toward the metricized statistics such as increasing their h-index, in order to sustain and progress on academic career path (Meyers and Quan, 2017). This has transformed science from an inquisitive driven exploration endeavor to a target-oriented enterprise and at the same time shifted the communication balance from diligent reporting to storytelling.

Reviewers, the pivotal stakeholders who ensure the quality and integrity of published results, work within a centralized, nontransparent, and nonincentivized voluntary system. In the context of modern research, science has evolved from a singular laboratory-based endeavor toward a multidisciplinary and geographically fragmented enterprise, where this voluntary system has now become unsustainable and unfair. It is unsustainable because reviewers do not receive any resources from the journals or their workplace to reproduce the results, and in the context of multidisciplinary research, individually they may not hold expertise or specialisation in all the related disciplines underpinning the data. It is unfair because reviewers do not get any reward, incentive, or recognition for their work. Although initiatives like Publons (Publons) have started a recognition system for reviewers, such recognition does not allow reviewers to have their fair share of the currency of science, publication. In general, reviewers undertake this laborintensive and critical work based on a vague idea of "academic service" in an assumed environment where academics have excellent job security with an ample amount of free time and resources.

According to Deutsche Bank's 2005 report, journals work in a "triple-pay" system where "the state funds most research, pays the salaries of most of those checking the quality of research, and then buys most of the published product" (Buranyi, 2017). Through such minimal production cost system, journals yield a profit margin as high as $36 \%$ dwarfing the profit margins of many other similar industries, while simultaneously owning the copyright of the published work (with exception of few open access journals).

Given the present cultural context of the publication industry, this paper proposes a blockchain and smart contract enabled peer-to-peer (P2P) publication model where publications will be incremental and authorship will be accumulative and shared with reviewers. This conceptual model is inspired by the transformational journey the music publishing industry has undertaken as it traverses from the vinyl age to Spotify age. Currently, a new music ecosystem is emerging, where instead of a whole album, music is published and sold as singles and artists get paid per stream or download. Artists are more inclined to build an incremental album with singles by engaging fans throughout the publication process via social media. Artist such as Grammy award winner Imogen Heap is publishing her music through a project called "Mycelia" (Mycelia) which utilizes blockchain and smart contract technologies. This project ensures that revenues for singles are automatically collected from steaming and derivatives like ringtone downloads, stage performances, and other royalty generating activities. The collected revenue is shared with all corresponding stakeholders (singer, song writer, composer, etc.), according to the percentage defined in the smart contract. Like music publishing, unit-wise academic publication has also been proposed by the Octopus project (Freeman, 2019) where full publication is broken down into 8 shorter units, scientific problem, hypothesis, method/protocol, results/data, analysis, interpretation, implementation, and review. Here, publication of each unit needs to be linked to at least one "above it" in the chain (except the review unit), and once completed, each unit will be published instantly for open review and rating by the reader through open repository like arXiv.org. 


\section{P2P PUBLICATION MODEL}

Utilizing blockchain and smart contract technology, the proposed $\mathrm{P} 2 \mathrm{P}$ publication model aims to bring two fundamental changes to the current publication model. First is the change from complete full-cycle publication to incremental unit-wise publication. Second is the introduction of a transparent and equitable recognition, rewarding, and responsibility-sharing mechanism between researchers and reviewers. For the P2P publication model, individual journal or publishing companies (e.g., Frontiers) will form a consortium-based private-permissioned blockchain network. This will have three key advantages: 1) it helps exclude predatory journals, 2) it helps easily identify duplicate and simultaneous submissions, and 3) it helps access to a cross-discipline pool of reviewers from multiple journals. Even though blockchain advocates openness, a consortium-led approach suits the need to exclude predatory journals (Cobey et al., 2018) from joining the network, which exploits the researchers (Cobey, 2017). Universities, research institutes, and companies alike can voluntarily join the consortium and operate within the network to further strengthen the network.

Simultaneous and duplicate publications, often withdrawn after receiving reviewers' feedback, result in waste of time and resources, for both reviewers and journals (Morse, 2007). Since blockchain provides an indisputable log of events, the journals or publishing companies, forming a network at a global scale, would be able to detect multiple simultaneous submissions or at least have an indisputable log of previous submission. Furthermore, a consortium of journals from different disciplines can grant access to a cross-discipline pool of reviewers.

A reputation profile will be issued for researchers and reviewers at the time of joining the network, both as a researcher and reviewer, namely, Res reputation and Rev reputation. The profile score will fluctuate dynamically according to the earning/deduction of reputation during their lifetime of operating on that journal-specific network. Reputation is a critical component of a digital economy and projects like the "educational reputation currency, Kudos" introduced by Open University (Sharples and Domingue, 2016) have already proposed the idea of generating educational currency using blockchain linked with academic achievements and credits. This educational currency will underpin the authenticity of the achievements, thereby making the trade of academic achievements more transparent and efficient.

The P2P publication model will also capitalize on recent initiatives like preregistration or registered report that enables researchers to preapprove their experimental design with the promise to get the paper published irrespective of the outcome (Chambers et al., 2014). Such initiatives will eventually alleviate the pressure of publishing "good results" and may also minimise the growing occurrence of "p-hacking" (data analysis manipulation) and or "HARKing" (hypothesizing after knowing the results). However, the preregistration model is based on the traditional full-cycle theme where only completed experiments will get published. The P2P model will combine the preregistration concept with the unit-wise publishing concept advocated by Octopus project (Freeman,
2019) and tools like ProtocolNavigator (Khan et al., 2014), Protocols.IO (Teytelman, 2018), and ISA (RoccaGSerra and Brandizi, 2010). Through these tools, researchers can document their experimental design and methodology in graphical and textual format and submit it for preregistration with identifiable units. Both journal and researchers agree on the objectives and expected outcome of each unit and the agreement will be codified into a smart contract along with the apportion of authorship between researchers and reviewers, along with the journal fees, credits/acknowledgment of other stakeholders such as industry partners or funding bodies, and data policies.

For researchers, reviewers, and readers, the $\mathrm{P} 2 \mathrm{P}$ publication model introduces a scoring-based schema. Upon successful completion of a unit of work for a preregistered experiment, the researcher will upload their work to a decentralized storage system such as IPFS and broadcast a transaction T on the journalspecific blockchain network, which includes the metadata of the research publication. Subsequently, the journal will be notified and after the preliminary editorial review, the smart contract will be invoked. Unit $t_{\text {score }}$ for that unit of work will be reset to zero and $\mathrm{N}$ reviewers will be invited to review the work. The smart contract will allocate a total of $\lambda$ percent of authorship to the reviewers, giving each reviewer a $\lambda / N$ percentage of authorship for that unit, regardless of the outcome of the submitted work (see Figure 1). This will encourage the reviewers to act in good faith as rejecting or accepting the submitted work bears no difference in benefits.

Like the traditional approach, each reviewer will review the work and provide feedback to the journal and researcher in a transparent manner, along with their affirm decision. In addition to the feedback and decision, the reviewers will also provide a satisfaction score $S$ for the reviewed work, which will be used to apportion the authorship share between researcher and reviewer(s). This step can be iterative if the feedback is for modification and improvement purposes. For each iteration,

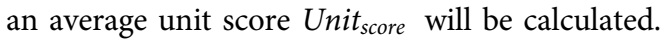

$$
\text { Unit }_{\text {score }}=\frac{\sum_{n=1}^{N} S_{n}}{N}
$$

This iterative feedback will facilitate researchers to improve the quality of their work and averaging the unit score at each iteration will safeguard researchers from any form of reviewers' bias or abuse. Once the reviewers reach a consensus on the acceptance/rejection decision, the smart contract will publish the unit of work along with a feedback thread. Each reviewer will be allocated an equal Rev $v_{\text {unit_score }}$ accordingly

$$
R e v_{\text {unit_score }}=\lambda\left(\text { Unit }_{\text {score }}\right) / N \text {. }
$$

For example, in the case of a unit that has $20 \%$ reviewer's share of authorship and from three reviewers received an average of 8.5 Unit $_{\text {score }}$, each reviewer will get Rev $_{\text {unit_score }}$ of 0.57 [(0.20 $\left.{ }^{\star} 8.5\right) / 3$ ].

Since reviews vary significantly in length and depth, it is important that the reviewers are rewarded fairly for their contribution. The value of reviewer's feedback is gauged by allowing readers to rate the reviewer's comments, recorded as 


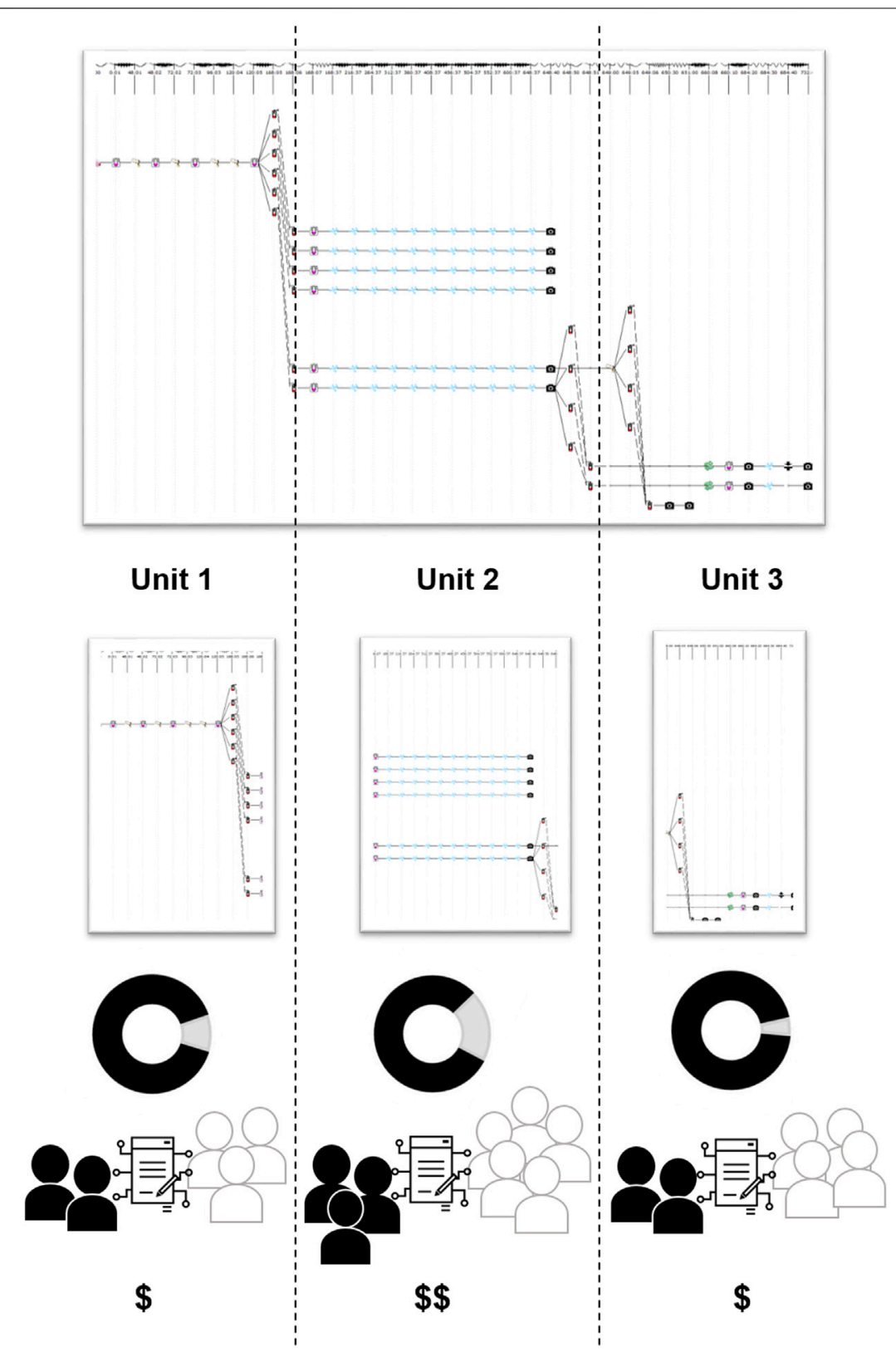

FIGURE 1 |P2P publication model. Preregistered experimental design encoded with ProtocolNavigator (top panel) is divided into three units (middle panel). Smart contract determines the shared authorship for each unit (bottom panel) between researchers (dark fill) and reviewers (no fill) along with journal fee for each unit (dollar sign).

a documentary of the work. This rating range $R$ (with positive/ negative value) will help to gauge reader's perception toward the rigor of individual reviewer's feedback and will be used as a weight to calculate individual reviewer's reputation, $R e v_{\text {reputation }}$. Even though initially each reviewer was allocated a $\lambda / N$ share in ownership, the readers eventually decide the reviewer's stake in the published unit, based on their contribution to the review process.

To ensure fair usage of the system and avoid spams, we propose that only the readers registered with the network should be allowed to rate the reviewer's work. Similar to the reviewers and researchers, readers will be assigned a reputation score which will change over time based on the reader's contribution to the network. A fraction of reader's repute $r$ can be used to determine the weightage of the rating $R$. Readers with higher community contribution will have a higher weight $\delta$ on the reviewer's score as compared to a reader with little or no contribution to the publication network. This will create a community-governed ecosystem such as the likes of Reddit and Stackoverflow. 


$$
R e v_{\text {reputation }}=R e v_{\text {unit_score }}\left(\frac{\sum_{i=1}^{n}\left(R * r^{\delta}\right)_{i}}{n}\right)
$$

The $R e v_{\text {reputation }}$ of the $\mathrm{N}=3$ reviewers, having $R e v_{\text {unit_score }}$ of 0.57 , receiving average $\mathrm{R}$ rating of $-1,0.5$, and one can be calculated as Reviewer1: $0.57 \times-1=-0.57$, Reviwer2: $0.57 \times$ $0.5=0.285$, and Reviewer3: $0.57 \times 1=0.57$, respectively. It can be clearly seen from the example above that the neutral readers can severely impact the overall share or contribution score of the reviewers, encouraging them to provide constructive and helpful feedback while simultaneously discouraging them to sign up for the review process with a sole objective of gaining $R e v_{\text {reputation }}$.

This social assessment approach can minimise or potentially eliminate the current asymmetrical decision-making environment where critical and trivial reviews often bear the same recognition (Zeeshan et al., 2018). This will also help readers to get a critical understanding of the unit of work and may form the basis of a crowdsourced double reviewing approach (reviewing the reviewers). This $R e v_{\text {reputation }}$ score will evolve as more readers read and rate that unit of publication, causing the reputation of the individual reviewer to increase or decrease over time. This dynamic feature of the algorithm will make the reviewer accountable for the lifetime of their review transaction history. In the case of the MMR-autism vaccine paper, if after 12 years, the publication is retracted, readers can revisit and update the feedback and rating. To avoid collusion and conflict of interest, the participants of a current "research transaction" (reviewers, researchers, and other stakeholders mentioned in the smart contract) will be excluded from the review or from scoring each other's work for the next $\mathrm{N}$ units of work.

The smart contract will also assign Res unit_score $_{\text {to }}$ tospective researchers involved with that unit.

$$
\text { Res }_{\text {unit_score }}=\text { Unit }_{\text {score }}-\lambda\left(\text { Unit }_{\text {score }}\right) .
$$

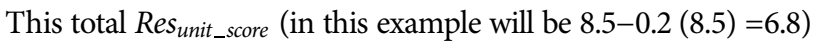
will be further divided within the contributing researchers according to their percentage of contribution as defined by the smart contract for that unit.

For researchers, a separate score, $\mathrm{Cit}_{\text {unit_score }}$ that captures direct and indirect citations of the publication, will be created. This also incentivizes researchers to collect rewards by publishing high-impact work on the proposed network and built reputation in an indirect way instead of the current direct citation metric. The equation below represents the Cit $_{\text {unit_score }}$ in the form of direct $\sigma$ and indirect citations $\Phi$. For indirect citations, a cap (e.g., three levels deep) can be introduced in the smart contract.

$$
C i t_{\text {unit_score }}=\sum_{i=0}^{n} \sigma_{i}+\sum_{j=0}^{n} \Phi_{j}
$$

A publication with 10 direct and 50 indirect citations can have Cit $_{\text {unit_score }}$ of 12.5 (indirect citations having a smaller contribution to the $\mathrm{Cit}_{\text {unit_score }}$ ). Like reviewer's reputation, researcher's reputation will also be weighted by the number of direct and indirect citations and dynamically updated over the lifetime of the journal.

$$
\text { Res }_{\text {reputation }}=\text { Res }_{\text {unit_score }}+\text { Cit }_{\text {unit_score }} .
$$

Finally, following the same example, Res $s_{\text {reputation }}$ can be calculated as $6.8+12.5=19.3$.

In case of a retraction or similar negative event, the smart contract will automatically nullify associated $\operatorname{Res}_{\text {unit_score }(s)}$ and Cit $t_{\text {unit_score }(s)}$, which will be deducted from the overall Res reputation of that individual researcher.

\section{INCENTIVIZATION AND RETRIBUTION IN P2P PUBLICATION MODEL}

Dynamic Res $s_{\text {reputation }}$ and Rev reputation scoring mechanisms introduced by P2P model incorporate the philosophy of proof of reputation (PoR) consensus protocols in blockchain technology (Shahaab et al., 2019), where the reputation of the validator is put at stake (incur a significant fine if they attempt to cheat) for validating each transacting block. In P2P model, researcher's and reviewer's reputations are augmented by quality work and diminished by malpractice. Furthermore, incremental publishing will facilitate researchers to build a complex dynamic portfolio which is comprised of Res reputation, Rev reputation, publishing journal, percentage of authorship, the type of unit, and frequency of review. This approach will safeguard researchers from the pitfall of "publish or perish" as they will be able to incrementally build up different components of their portfolio to sustain and advance their academic career. The cultural impact of alleviating these burdens from researchers is hard to foresee at this point but it is almost inevitable that these mechanisms will increase creativity and endorsement of creativity at a granular level and in incremental fashion that can revert science to an exploration-based endeavor, even at an enterprise scale.

By sharing authorship with reviewers, the P2P model will fundamentally change the incentive as well as the engagement approach for reviewers. Although a number of blockchain-based initiatives have been implemented that introduce different forms of reward mechanisms, for example, digital token for reviewers (Jan 2018) (Jan et al., 2018) (Zeeshan et al., 2018) (Niya et al., 2019), here we argue that incorporation of any form of financial incentive may have a negative impact on scientific research, as evident by the "cash for publication" incentive approach introduced for researchers about a decade ago (Fuyuno and Cyranoski, 2006). Such initiatives adopted by different countries and organizations are now proven to be "discriminatory, unscientific, and dangerous" (Bamji, 2019). In our proposed P2P model, the incentive is not financial but rather ownership. Rev reputation is directly linked with the ownership and proof of work (depth and breadth of feedback) and is weighted by reader-driven ratings of reviewers. Holding reviewers accountable for their review work will fundamentally change the quality of published research work, along with the researchers, who can see their reputation diminished through future debate, bad press, or retraction. Such a "carrot and stick" approach is a 
time-tested universal model that aligns with the human psyche (Ayres, 2010).

For journals, the P2P model will not contradict the current "triple-pay" system. Smart contracts will ensure that journal fees get automatically paid as each unit of work is validated and published on the network. Voluntary incorporation of universities and research institutions will enable journals to demonstrate their popularity in the scientific community. The more the institutes host networks, the more secure their integrity will become and the more authority the journals will gain within the scientific community.

\section{IMPACT ON RESEARCH REPRODUCIBILITY CRISIS}

The P2P publication model addresses two key sociocultural issues of the research reproducibility crisis, the stress researchers endure to progress their career and the required recognition and rewards for the reviewers' hard work. Beyond these two key issues, there are a number of sociocultural and philosophical issues associated with irreproducibility which are identified and addressed by the P2P publication model.

\section{Openness and Freedom}

Academic freedom usually refers to the flexibility of researchers to change study design. However, in the context of irreproducibility, this privilege is often exploited to get "good results" and subverts the openness to publish negative results, flawed design, and the rationale for design change. The P2P model sets a balance between openness and freedom by integrating the preregistration concept with unit-wise publication. For each unit, the smart contract will compel researchers to publish all experimental details and results in order to invoke review process. The immutability aspect of blockchain will also prevent researchers from "p-hacking" and or "HARKing." While ensuring openness and data integrity, the P2P model simultaneously ensures freedom and promotes researchers to pursue their curiosity-a fundamental tenant of science. After the publication of each unit, the researcher has the flexibility to change the course of progression by registering new units of work replacing previous work without any impact on their academic portfolio. Under traditional publication models (including the preregistration model), researchers are restricted to pursue their curiosity because they need to complete the whole experiment and all publication steps, despite feeling disengaged. Publishing scientific work just for the sake of an academic portfolio is demoralizing for the researchers and detrimental for science itself (Holyoke and Medawar, 1980).

\section{Diversity and Distance of Multidisciplinary Research}

Scientific research has become multidisciplinary, through the diversity of disciplinary collaborations and the sociocultural and geographical distance between data producers and consumers. Addressing this changing environment, discipline neutral journals like PLOS One and IEEE Access are evolving as multidisciplinary and open outlets. The P2P model will augment this trend through the ability to include unit-specific expertise for the review process. In addition to the opportunity of readers to rate reviewers, the $\mathrm{P} 2 \mathrm{P}$ publication model has the potential to forge a democratic citizen science environment by allowing the public to participate in the review process through open invitation to review the unit of work. Acquiring authorship percentages and engagement at an equal level with other academics will minimise the social and intellectual distance between academic and nonacademic citizens, which eventually will facilitate the shaping of science-informed public policies more efficiently and effectively. This may also facilitate overcoming the effect of groupthink on reproducibility. Groupthink is defined by Iring Janis, "a psychological drive for consensus at any cost that suppresses dissent and appraisal of alternatives in cohesive decision-making groups." This tendency deters like-minded academics from rigorous review, particularly when the results confirm the professional audience's preconceptions (Kuklick and Janis, 1973). Groupthink also deters academics from communicating and engaging with the public about ongoing research, despite the fact that most of the researches are publicly funded. The $\mathrm{P} 2 \mathrm{P}$ publication model will transform this mindset by valuing the public on par with academics' stakeholders. Citizen science projects like Foldit have demonstrated that game players with little or no biology background-solved structure of many proteins include an HIV enzyme within weeks, which scientists were struggling to solve for years (Khatib et al., 2010).

\section{LIMITATIONS AND FUTURE WORK}

Reputation is the core driver for this $\mathrm{P} 2 \mathrm{P}$ publication model where both researchers and reviewers will strive to improve their reputational score. Both parties' reputations are dynamic and are weighted by citation numbers (in case of researchers) and rating of readers (in case of reviewers). Despite the fact that safeguards are in place to detect and deter bad actors from manipulating researchers' or reviewers' reputation, malpractice is expected. For example, researchers may request colleagues, students, or friends to cite their research or reviewers to rate their review favorably in order to increase their reputation artificially. Reviewers may also be subjected to negative ratings from readers. In such scenarios, social network analysis (SNA) like algorithms can be utilized to detect collisions or manipulations.

The proposed P2P publication model can only function well and add significant value if it is adopted by masses, since it requires multiple journals to collaborate and researchers and reviewers from multidisciplines to participate. Bootstrapping such ecosystem at scale and gaining the confidence of academia will remain the biggest challenge for $\mathrm{P} 2 \mathrm{P}$ publication model. We strive to engage multiple stakeholders in building the proof of concept of the proposed P2P publication model and release the artifacts as an open-source project.

As an enhancement proposal, we would like to revisit the acceptance criteria and build them in the algorithm so that the 
publications are accepted automatically when the criteria are met. One possible choice could be using the publication score as a metric and allow publications only when the score is above a certain, dynamically adjusted threshold. However, this could create a positive feedback loop; therefore, we are carefully considering how to implement it in our prototype.

We aim to develop and deploy the full-scale P2P publication network on a permissioned distributed ledger such as Hyperledger or Corda, inviting academics and researchers to join the network and contribute to the success of the model, in a pan-Wales setting at the initial stage. An opportunity to bootstrap the initiative can be multidisciplinary research on COVID-19 with the potential of publishing the findings with universitybased journals of the network. Once the researchers and reviewers see a fair and transparent distribution of reward, we are certain that our proposed model will be adopted by the mainstream publishing houses.

\section{POST JOURNAL LANDSCAPE}

Science Europe in recent years introduced an initiative called "Plan S" that demands all publications to be open access and copyrights to be retained by the authors under a creative common open license like agreement (Else, 2018). The smart contract and indirect citation quantification ability of the P2P model can be adapted to safeguard this copyright issue. The $\mathrm{P} 2 \mathrm{P}$ publication model can also benefit emerging preprint repositories like bioRxiv (from Cold Spring Harbor) where researchers can post their manuscripts for free and get feedback from readers before submission to journals. However, if the P2P model is adopted by these repositories, instead of feedback, the unit-wise review process can be completed without the need for submission to journals. In this respect, the impact of eliminating "payment" from the publication process is hard to foresee, but it will definitely address some of the growing concerns regarding open access journals (Clare, 2018).

As science in the 21 st century is transforming into an open enterprise, an "intelligent openness" (Boulton et al., 2012)

\section{REFERENCES}

Ayres, I. (2010). Carrots and sticks: unlock the power of incentives to get things done. New York, NY: Bantam Dell Publishing Group, Div of Random House, Inc.

Baker, M. (2016). 1,500 scientists lift the lid on reproducibility. Nature 533, 452. doi:10.1038/533452a

Bamji, A. N. (2019). Cash for publication is discriminatory, unscientific, and dangerous. BMJ 13, 11915. doi:10.1136/bmj.11915

Begley, C. G., and Ellis, L. M. (2012). Drug development: raise standards for preclinical cancer research. Nature 483, 531. doi:10.1038/483531a

Boulton, G., Campbell, P., Collins, B., Elias, P., Hall, W., Laurie, G., et al. (2012). Science as an open enterprise. $R$. Soc. Available at: https://royalsociety.org/topicspolicy/projects/science-public-enterprise/report/ (Accessed June 21, 2012).

Brainard, J. (2018). What a massive database of retracted papers reveals about science publishing's “death penalty". Science 80 . doi:10.1126/science.aav8384

Buranyi, S. (2017). No Title. Guard. Available at: https://www.theguardian.com/ science/2017/jun/27/profitable-business-scientific-publishing-bad-for-science (Accessed June 27, 2017) framework is sought, that will ensure the integrity of data and knowledge through open accessibility and at the same time will also safeguard the privacy and intellectual property rights of individual scientists. The $\mathrm{P} 2 \mathrm{P}$ publication model proposed here addresses the challenges of "intelligent openness" through blockchain and smart contract-based technologies. In summary, it is believed that openness and equitability of the peer-review process proposed by this conceptual P2P publication model will introduce a new paradigm of communication, collaboration, competition, and creativity for researchers and reviewers.

\section{DATA AVAILABILITY STATEMENT}

The original contributions presented in the study are included in the article; further inquiries can be directed to the corresponding author.

\section{AUTHOR CONTRIBUTIONS}

IK conceptualized the model and AS implemented the mathematical basis of the model. Both IK and AS wrote the manuscript.

\section{FUNDING}

This research has been supported by Knowledge Economy Skills Scholarships (Scholarship \#CMK219) a major pan-Wales operation supported by the European Social Funds through the Welsh Government.

\section{ACKNOWLEDGMENTS}

The authors would like to thank Dr. Simon Thorne for proofreading the manuscript.

Chambers, C. D., Feredoes, E., Muthukumaraswamy, S. D., and Etchells, P. J. (2014). Instead of "playing the game" it is time to change the rules: registered Reports at AIMS Neuroscience and beyond. AIMS Neurosci. 1 (1), 4-17. doi:10. 3934/Neuroscience.2014.1.4

Chambers, C. D. (2019). The battle for reproducibility over storytelling. Cortex, 113, A1-A2. doi:10.1016/j.cortex.2019.03.009

Clare, H. (2018). Considering the implications of the finch report. Available at: https://scholarlycommunications.jiscinvolve.org/wp/2018/10/22/open-accessbriefing-paper-considering-the-implications-of-the-finch-report/ (Accessed October 22, 2018).

Cobey, K. D., Lalu, M. M., Skidmore, B., Ahmadzai, N., Grudniewicz, A., and Moher, D. (2018). What is a predatory journal? A scoping review. F1000Res 7. 1001. doi:10.12688/f1000research.15256.2

Cobey, K. (2017). Illegitimate journals scam even senior scientists. Nature 549, 7. doi:10.1038/549007a

Eggertson, L. (2010). Lancet retracts 12-year-old article linking autism to MMR vaccines. CMAJ 182, E199. doi:10.1503/cmaj.109-3179

Else, H. (2018). Radical open-access plan could spell end to journal subscriptions. Nature 561, 17. doi:10.1038/d41586-018-06178-7 
Errington, F., Dimaio, F., DiMaio, F., Cooper, S., Kazmierczyk, M., Gilski, M., et al. (2011). Crystal structure of a monomeric retroviral protease solved by protein folding game players. Nat. Struct. Mol. Biol. 18, 1175. doi:10.1038/nsmb.2119

Freedman, L. P., Cockburn, I. M., and Simcoe, T. S. (2015). The economics of reproducibility in preclinical research. PloS Biol. 13, e1002165. doi:10.1371/ journal.pbio.1002165

Freeman, A. (2019). Octopus: a revolution in scientific publishing. BMJ Evid Based Med. 24, A29-A30. doi:10.1136/bmjebm-2019-EBMLive.56

Fuyuno, I., and Cyranoski, D. (2006). Cash for papers: putting a premium on publication. Nature 441, 792. doi:10.1038/441792b

Holyoke, T. C., and Medawar, P. B. (1980). Advice to a young scientist. Antioch Rev. 38, 259. doi:10.2307/4638313

Hutchinson, L., and Kirk, R. (2011). High drug attrition rates-where are we going wrong? Nat. Rev. Clin. Oncol. 8, 189. doi:10.1038/nrclinonc.2011.34

James, H. (2020). The Lancet has made one of the biggest retractions in modern history. How could this happen? Guard. Available at: https://www.theguardian. com/commentisfree/2020/jun/05/lancet-had-to-do-one-of-the-biggest-retractionsin-modern-history-how-could-this-happen (Accessed June 5, 2020).

Jan, Z. (2018). "Recognition and reward system for peer-reviewers," in CEUR workshop proceedings, Aachen, Germany, March 2018.

Jan, Z., Third, A., Ibanez, L. D., Bachler, M., Simperl, E., and Domingue, J. (2018). "ScienceMiles: digital currency for researchers," in The web conference 2018companion of the world wide web conference, Lyon, France, April 23-27, 2018.

Khan, I. A., Fraser, A., Bray, M. A., Smith, P. J., White, N. S., Carpenter, A. E., et al. (2014). ProtocolNavigator: emulation-based software for the design, documentation and reproduction biological experiments. Bioinformatics 30, 3440. doi:10.1093/bioinformatics/btu554

Krzywda, P. (2013). The excel depression. The New York Times. Available at: https://www.nytimes.com/2013/04/19/opinion/krugman-the-excel-depression. html (Accessed April 19, 2013).

Kuklick, B., and Janis, I. L. (1973). Victims of groupthink: a psychological study of foreign-policy decisions and fiascoes. J. Am. Hist. 60, 857. doi:10.2307/1917768

Meyers, M. A., and Quan, H. (2017). The use of the h-index to evaluate and rank academic departments. J. Mat. Res. Technol. 6, 304. doi:10.1016/j.jmrt.2017.09.004

Morse, J. M. (2007). Duplicate publication. Qual. Health Res. 17, 1307-1308. doi:10.1177/1049732307309159

Mycelia. Available at: http://myceliaformusic.org/.
Niya, S. R., Pelloni, L., Wullschleger, S., Schaufelbühl, A., Bocek, T., Rajendran, L., et al. (2019). "A blockchain-based scientific publishing platform,"in ICBC 2019 IEEE international conference on blockchain and cryptocurrency, Seoul, Korea, May 14-17, 2019. doi:10.1109/BLOC.2019.8751379

Organisation, W. H. (2019). Measles-European region. Available at: https://www. who.int/csr/don/06-may-2019-measles-euro/en/ (Accessed May 6, 2019).

Publons. Available at: https://publons.com.

RoccaGSerra, P., and Brandizi, M. (2010). ISA software suite: supporting standardscompliant experimental annotation and enabling curation at the community level. Bioinformatics 26 (18), 2354G2356. doi:10.1093/bioinformatics/btq415

Shahaab, A., Lidgey, B., Hewage, C., and Khan, I. (2019). Applicability and appropriateness of distributed ledgers consensus protocols in public and private sectors: a systematic review. IEEE Access. 7, 43622. doi:10.1109/ACCESS.2019.2904181

Sharples, M., and Domingue, J. (2016). "The blockchain and kudos: a distributed system for educational record, reputation and reward," in 11th European Conference on Technology Enhanced Learning, Lyon, France, September 13-16, 2016.

Teytelman, L. (2018). No more excuses for non-reproducible methods. Nature 560, 411. doi:10.1038/d41586-018-06008-w

Watkins, J., Wulaningsih, W., Da Zhou, C., Marshall, D. C., Sylianteng, G. D. C., Dela Rosa, P. G., et al. (2017). Effects of health and social care spending constraints on mortality in England: a time trend analysis. BMJ Open. 7, e017722. doi:10.1136/bmjopen-2017-017722

Zeeshan, J., Third, A., Bachler, M., and Domingue, J. (2018). "Peer-reviews on the blockchain," in 1st Workshop on Reframing Research (RefResh), Cologne, Germany, December 5-7, 2018.

Conflict of Interest: The authors declare that the research was conducted in the absence of any commercial or financial relationships that could be construed as a potential conflict of interest.

Copyright (c) 2021 Khan and Shahaab. This is an open-access article distributed under the terms of the Creative Commons Attribution License (CC BY). The use, distribution or reproduction in other forums is permitted, provided the original author(s) and the copyright owner(s) are credited and that the original publication in this journal is cited, in accordance with accepted academic practice. No use, distribution or reproduction is permitted which does not comply with these terms. 